\title{
Diagnóstico de enfermagem para pacientes portadores do COVID-19
}

\author{
Nursing diagnosis for patients with coronavirus. COVID-19
}

Diagnóstico de enfermería para pacientes con COVID-19

Sandra Conceição Ribeiro Chícharo

ORCID: http://orcid.org/0000-0002-1487-0088

Universidade Federal Fluminense, Brasil Centro Universitário São José, Brasil

E-mail: sandracrichicharo@gmail.com

Cristiane Teles Frazão

ORCID: https://orcid.org/0000-0003-0185-3470 Centro Universitário São José, Brasil E-mail: crisfrazao.ct@gmail.com

Juliana Soares de Souza Nogueira

ORCID: https://orcid.org 0000-0001-7850-1719 Centro Universitário São José, Brasil

E-mail: jnogrjenf@gmail.com

Gabrielli dos Santos Netto de Melo

ORCID: https://orcid.org/0000-0002-5095-6671 Centro Universitário São José, Brasil

E-mail: gaabinmelo@gmail.com

Gabriel Silva da Rosa de Carvalho

ORCID: https://orcid.org/0000-0001-5539-7752 Centro Universitário São José, Brasil

E-mail: gabrielsilvarosa98@gmail.com

Dilson da Silva Lopes

ORCID: https://orcid.org/0000-0003-4652-710X Centro Universitário São José, Brasil

E-mail: dilsonenfermagem2016@gmail.com

Washington Luiz Raimundo Gomes Tavares

ORCID: http://orcid.org/0000-0001-9195-7999 Centro Universitário São José, Brasil

E-mail: ton.luiz@outlook.com

\begin{abstract}
Resumo
Um surto mundial de síndrome respiratória aguda grave (SARS) foi associado a exposições originárias de um único profissional de saúde da província de Guangdong, na China. Objetivos: Analisar quais os principais diagnósticos de enfermagem mais predominantes aos pacientes com COVID-19, mediante sua sintomatologia clássica. Metodologia: Revisão integrativa da literatura feita nas bases de dados Pubmed, Lilacs e Medline com recorte temporal dos últimos 12 anos. Resultados: Dentre a sintomatologia descrita nos artigos selecionados, houve predominância dos distúrbios do trato respiratório e as lesões cardiovasculares disseminadas que corroboraram para validação das taxonomias. Conclusão: O que representa a enfermagem mediante a esta patologia. A enfermagem e a responsável pelos cuidados 24 horas por dia dos indivíduos eferente aos seus cuidados, necessidades e anseios. A enfermagem monta uma parte importante no que se refere o cuidar, $\mathrm{n}$ maneira geral, tano físico, mental e espiritual, usando os diagnósticos iniciais referidos por sintomas e análise de casos e referencias clinicas.
\end{abstract}

Palavras-chave: COVID-19; SARS-COV1; SARS-CoV-2; Enfermagem; Diagnóstico de enfermagem.

\begin{abstract}
A worldwide outbreak of severe acute respiratory syndrome (SARS) has been associated with exposures from a single health professional in Guangdong province, China. Objectives: To analyze which are the main nursing diagnoses most prevalent to patients with COVID-19, based on their classic symptoms. Methodology: Integrative literature review carried out in the Pubmed, Lilacs and Medline databases with a time frame of the last 12 years. Results: Among the symptoms described in the selected articles, there was a predominance of respiratory tract disorders and disseminated cardiovascular lesions that corroborated for the validation of taxonomies. Conclusion: What nursing represents through this pathology. Nursing is responsible for the 24-hour care of individuals that is relevant to their care, needs and desires. Nursing plays an important part in terms of caring, in general, physical, mental and spiritual, using the initial diagnoses referred to by symptoms and analysis of cases and clinical references.
\end{abstract}

Keywords: COVID-19; SARS-COV1; SARS-CoV-2; Nursing; Nursing diagnosis. 


\begin{abstract}
Resumen
Un brote mundial de síndrome respiratorio agudo severo (SARS) se ha asociado con exposiciones de un solo profesional de la salud en la provincia de Guangdong, China. Objetivos: Analizar los principales diagnósticos de enfermería más prevalentes a los pacientes con COVID-19, en base a sus síntomas clásicos. Metodología: Revisión integrativa de la literatura realizada en las bases de datos Pubmed, Lilacs y Medline con un marco temporal de los últimos 12 años. Resultados: Entre los síntomas descritos en los artículos seleccionados, hubo predominio de trastornos del tracto respiratorio y lesiones cardiovasculares diseminadas que corroboraron para la validación de taxonomías. Conclusión: Qué representa la enfermería a través de esta patología? La enfermería es responsable del cuidado de las personas las 24 horas del día que sea relevante para su cuidado, necesidades y deseos. Enfermería juega un papel importante en el cuidado, en general, físico, mental y espiritual, utilizando los diagnósticos iniciales referidos por síntomas y análisis de casos y referencias clínicas.
\end{abstract}

Palabras clave: COVID-19; SARS-COV1; SARS-CoV-2; Enfermería; Diagnóstico de enfermeira.

\title{
1. Introdução
}

Um surto de um vírus bem complexo (SARS-CoV-2) foi relatado em dezembro de 2019 na Ásia, mas precisamente na China, na cidade de Wuhan (Província de Hubei), que se tornou o epicentro deste surto. Relacionado a uma nova cepa de coronavírus, até então não identificada em humanos, sendo responsável por causar uma infeção do trato respiratório inferior grave, "pneumonia de causa desconhecida", que rapidamente se espalhou para outras regiões da China, países asiáticos adjacentes, atingindo 6 continentes, incluindo 66 países, sendo responsável pela atual pandemia (Wu et al., 2020; Neeltje et al., 2020; Shohei et al., 2020; Bai, 2020).

Após ter o seu material genético isolado e identificado, o sequenciamento genômico e a análise filogenética evidenciou que o novo patógeno se tratava de um betacoronavírus, mesmo subgênero da síndrome da insuficiência respiratória aguda grave (SARS), envolvida na epidemia na China em 2003, e da síndrome respiratória do Médio Oriente (MERS), que atingiu o Oriente Médio em 2012. Inicialmente a Organização Mundial da Saúde denominou o vírus como "COVID-19" e posteriormente, recebeu o nome de SARS-CoV-2 (Severe Acute Respiratory Syndrome Coronavirus 2) pelo Comitê Internacional de Taxonomia de Vírus (Estrabelli \& Uip, 2020; Yuen et al., 2020).

O novo coronavírus (COVID-19) associado à síndrome respiratória aguda grave/SARS (SARS-CoV-2) representa uma emergente ameaça à saúde mundial. Globalmente a população foi pela infecção, com aproximadamente 80.000 casos confirmados da doença e uma prevalência de óbito em torno de 2.700 ao redor do mundo no mês de fevereiro de 2020. (Matt $e t$ al., 2020; Yuen et al., 2020).

Existem certas peculiaridades quanto à referida patologia, principalmente em qual seria a origem do vírus. Acredita-se que o mercado atacadista de frutos do mar de Huanan é pelo menos um dos lugares onde SARS-CoV-2, proveniente de um animal desconhecido, deve ter ultrapassado a barreira entre espécies para infectar humano (Yuen et al., 2020). Por outro lado, estudos ressaltam que os coronavírus emergentes seriam originários de morcegos, representados por coronavírus SARS (SARS-CoV) e coronavírus MERS (MERS-CoV) (Hu et al., 2015).

O SARS-CoV-2 é um vírus zoonótico, de grupo de vírus de genoma de RNA simples de sentido positivo (Wertheim et al., 2013), altamente mutagênico cujas taxas de mutação são em ordem de uma por 1.000 a 10000 nucleotídeos replicados, com alta frequência de recombinação homóloga de RNA e possuem genomas maiores que permitem exacerbada plasticidade na acomodação e modificação de genes, e consequentemente maior variedade de cepas. Além disso, são capazes de se adaptar a novos hospedeiros e nichos ecológicos (Woo et al., 2009).

Pertence a ordem Nidovirales, da família Coronaviridae, conhecidos desde meados dos anos 1960. As cepas descritas até o momento são: alfa coronavírus HCoV-229E e alfa coronavírus HCoV-NL63 (Descoberto em 2004), beta coronavírus HCoV-OC43 e beta coronavírus HCoV-HKU1 (Descoberto em 2005), SARS-CoV (causador da síndrome respiratória aguda grave ou SARS), MERS-CoV (causador da síndrome respiratória do Oriente Médio ou MERS) e SARSCoV-2, um novo coronavírus descoberto no final do ano 2019 (Lima, 2020; Nobre et al., 2014). 
Os vírus atuam como agentes etiológicos predominantes em IRA. As manifestações clínicas graves associadas a doenças do trato respiratório inferior representam fatore de risco para indivíduos com comorbidades e doenças crônicas, tais como: cardiopatia, pneumopatia, diabetes, obesidade e asma. (Nobre et al., 2014).

Além do acometimento do sistema respiratório, recentemente estudos apontaram a relação do coronavírus 2019 (2019-nCoV) em disfunções cardíacas e hematológicas (coagulopatia disseminada). Esses achados corroboram cada vez mais com a teoria de que o COVID-19 é uma patologia de evolução rápida e sistêmica (NING, 2020). Em função do crescimento acelerado da pandemia, a Organização Mundial da Saúde (OMS) declarou o CoVID-19 como uma "emergência de saúde pública de importância internacional” (Wu et al., 2020).

Nesse contexto, a enfermagem é a categoria profissional responsável pela assistência prestada aos pacientes infectados pelo novo coronavírus em ambiente hospitalar, prestando cuidados de forma integral, 24 horas por dia, além de apoio emocional. Sendo atribuição do enfermeiro, realizar o diagnóstico de enfermagem conforme os sintomas evidenciados e traçar um plano de cuidados aplicados a cada paciente e de acordo com as suas necessidades.

\section{Metodologia}

Trata-se de uma pesquisa qualitativa reveladora da realidade atual dos diversos sintomas em indivíduos acometidos pelo Covid-19. Métodos este relevante para dar suporte a interpretação do pesquisador quanto as sintomatologias mais evidenciadas na infecção pelo SARS-CoV-2. A fim de traçar um diagnóstico preciso e intervenções de enfermagem adequadas acerca da assistência a esses pacientes. além de favorecer uma maior compreensão quanto ao fenômeno estudado (Pereira et al., 2018). O presente estudo foi elaborado a partir de uma revisão da literatura com a finalidade de fundamentar teoricamente um determinado objetivo. Permitindo adquirir e atualizar o conhecimento a partir de resultados de artigos já divulgados por outros autores sobre a temática "Diagnóstico de enfermagem para pacientes portadores do COVID-19", em um curto espaço de tempo, de modo a incorporar a aplicabilidade sobre o que responde ao objetivo do trabalho.

O presente estudo foi elaborado a partir de uma revisão da literatura com a finalidade de fundamentar teoricamente um determinado objetivo. Permitindo adquirir e atualizar o conhecimento a partir de resultados de artigos já divulgados por outros autores sobre a temática "Diagnóstico de enfermagem para pacientes portadores do COVID-19", em um curto espaço de tempo, de modo a incorporar a aplicabilidade sobre o que responde ao objetivo do trabalho. Trata-se de uma pesquisa qualitativa reveladora da realidade atual dos diversos sintomas em indivíduos acometidos pelo Covid-19. Métodos este relevante para dar suporte a interpretação do pesquisador quanto as sintomatologias mais evidenciadas na infecção pelo SARSCoV-2. A fim de traçar um diagnóstico preciso e intervenções de enfermagem adequadas acerca da assistência a esses pacientes. além de favorecer uma maior compreensão quanto ao fenômeno estudado (Pereira et al., 2018).

O levantamento bibliográfico fundou-se em materiais indexados em fontes de informação eletrônica (artigos científicos publicados em revista e periódicos) utilizando as seguintes bases de dados: National Center for Biotechnology Information - Pubmed (https://www.ncbi.nlm.nih.gov/pubmed); Literatura Latino-Americana e do Caribe em Ciências da Saúde - Lilacs; Scientific Eletronic Library; Scielo (http://scielo.br/); Medical Literature Analysis and Retrieval System Online - Medline; Portal Regional da BVS (Biblioteca Virtual em Saúde), Ministério de saúde - MS e revistas eletrônicas.

A seleção das produções científicas ocorreu de acordo com seguintes os critérios de inclusão: textos publicados em português e inglês, que apresentassem estreita relação com o referido tema, com recorte temporal nos últimos 12 anos, no período de 2009 a 2020. Utilizando as seguintes palavras-chaves: SARS-COV1; SARS-CoV-2; Enfermagem e Diagnóstico de enfermagem. E de exclusão: artigos científicos publicados em outros idiomas e que não estabeleceram relação direta com o tema proposto. 
O estudo ainda buscou identificar as sintomatologias predominantemente descritas pelos autores em suas publicações, com a finalidade de traçar os diagnósticos de enfermagem (DE) para os pacientes acometidos pela infecção pelo coronavírus, de acordo com os sistemas de classificação de enfermagem na construção DE, relacionando-os a estratégias taxonômicas propostas pela NANDA-I (North American Nursing Diagnosis Association International), a qual classifica os diagnósticos de enfermagem em domínios e classes, constituídos por título, com uma definição conceitual, características definidoras e fatores relacionados ou de risco, sendo mundialmente o mais utilizado e difundido. Intervenções de Enfermagem, pela Intervention Classification (NIC) e os resultados de enfermagem, pela Nursing Intervention Classification (NOC), aplicados a estes pacientes (Nanda, 2018-2010; Pereira, 2010).

\section{Resultados}

Baseados nas publicações analisadas, os sinais e sintomas mais comuns relatados em função da infecção pelo SARS-CoV-2 foram: febre, tosse seca, fadiga, expectoração, dor de garganta, odinofagia e rinorreia, cefaleia, mialgia ou artralgia, calafrios, náuseas ou êmese, congestão nasal, diarreia, hemoptise e congestão conjuntival. Além de dispneia, taquipnéia, sinais de esforço respiratório, sangramento pulmonar, hipotensão, cianose, descompensação das doenças de base, linfopenia e insuficiência renal, requerendo internação hospitalar (Cespedes \& Souza, 2020; Lima, 2020; MS, 2020).

Um estudo realizado em Wuhan apontou a sepse decorrente da covid-19 como sendo a complicação mais prevalente em praticamente em $100 \%$ dos pacientes que evoluíram para óbito e $42 \%$ daqueles que sobreviveram à doença, seguida de insuficiência respiratória. Os casos de sepse e choque séptico comumente estão associados à coagulação intravascular disseminada e que é capaz de elevar o dímero-D e alteração das enzimas hepáticas AST e ALT. Propondo neste contexto como diagnósticos de enfermagem: risco de choque, risco de tromboembolismo venoso, risco de função hepática prejudicada (BBC NEWS, 2020).

\section{Discussão}

Estudos realizados acerca das patologias causadas pela infecção por SARS-CoV-2 pode estar relacionado a ECA2. Demonstrando que a síndrome respiratória aguda (SARS-CoV-2) infecta as células do hospedeiro através dos receptores ECA2, uma vez que é desencadeada pela ligação da espícula proteica do vírus na ECA2, a qual é altamente expressa no coração e nos pulmões, causando a doença por coronavírus (COVID-19) relacionada à pneumonia, comprometendo os pulmões em função do acúmulo progressivo de detritos e fluidos, provocando síndrome do desconforto respiratório agudo (SDRA) (Zheng, 2020).

Sua principal característica é causar uma lesão pulmonar, provocando excesso de fluidos tanto no interstício como nos alvéolos, Com dano alveolar difuso, com liberação de citocinas pró-inflamatórias, como fator de necrose tumoral, interleucina IL -1, IL-6 e IL-8, que danificam o endotélio capilar e o epitélio alveolar. Cujas consequências incluem prejuízo na troca gasosa, diminuição da complacência pulmonar e aumento da pressão arterial pulmonar (Siegel, 2020).

A enzima conversora de angiotensina 2 (ECA2) é uma aminopeptidase que exerce papel vital no bom funcionamento do coração, bem como, desenvolvimento de doenças crônicas como hipertensão e diabetes mellitus. Portanto, o mecanismo lesão aguda no miocárdio e danos crônicos ao sistema cardiovascular, pode estar relacionado a ECA2. Esta enzima é altamente expressa na boca e na língua, o que propicia a entrada do vírus (Cespedes \& Souza, 2020; Zheng et al., 2020). Acredita-se a cascata de citocina proveniente de um desequilíbrio da resposta das células T auxiliar dos tipos 1 e 23,6, associado a disfunção respiratória e hipoxemia causada pelo COVID-19, atuem como fatores desencadeador de danos a células do miocárdio (Zheng, 2020). 
Um estudo publicado na The Lancet relacionou valores séricos elevados de interleucina-6 (IL-6), troponina 1 ultrasensível e DHL aos casos de pacientes que evoluíram para óbito. Sugerindo que em pacientes com COVID-19, a incidência de sintomas respiratórios e cardiovasculares é alta, devido à resposta inflamatória sistêmica e a desordens do sistema imune durante a progressão da doença (Zhou et al., 2020).

Um outro estudo realizado por Bangalore et al (2020) evidenciou que pacientes com Covid-19 que apresentavam supradesnivelamento do segmento ST, indicando potencial infarto agudo do miocárdio, com alta prevalência de doença obstrutiva. Corroborando com achados em estudo semelhante desenvolvido por Mohammad et al. (2020) que evidenciou Síndrome coronariana aguda e infarto do miocárdio foram posteriormente à SARS.

A coagulopatia induzida por sepse, também foi relatada dentre as manifestações clínicas da infecção pelo cornavírus. Em que pacientes com COVID-19 apresentaram hipercoagulabilidade decorrente da alteração de células endoteliais induzida pelo processo infeccioso resultando em excesso de produção de trombina e menor fibrinólise. Ademais, concomitantemente ao quadro de hipóxia grave estimulando trombose por aumento da viscosidade sanguínea e sinalização dependente de fator de transcrição induzida pela diminuição das taxas de oxigênio no sangue. Tendo como consequência a oclusão e formação de microtromboses em pequenos vasos pulmonares de pacientes críticos com COVID-19 (NING et al., 2020).

Diante da problemática explanada e baseado nos resultados das pesquisas analisadas, foram selecionados as sintomatologias predominantemente encontradas nos pacientes acometidos pelo COVID-19, e realizado o diagnóstico de enfermagem a partir do NANDA, intervenções de enfermagem - NIC e resultado esperado - NOC, verificamos a existência da exacerbação sintomatológica da patologia em sequencia de 3 sistemas orgânicos fisiológicos, na qual são eles o sistema cardíaco, sistema respiratório e sistema hematológico.

O diagnóstico inicial foi traçado a partir dos sinais e sintomas evidenciados de forma majoritária durante a análise das pesquisas. Utilizando como referência - Diagnóstico de enfermagem da NANDA - 1, definições e classificação. Mediante a análise dos diagnósticos estudados chegamos na identificação de XX diagnósticos que podem ser empregados no plano de ação de assistência de enfermagem, conforme podemos observar nas figuras 1, 2 e 3 abaixo.

Figura 1. Lista de Diagnósticos associados aos COVID-19, domínios 1, 2 e 3.

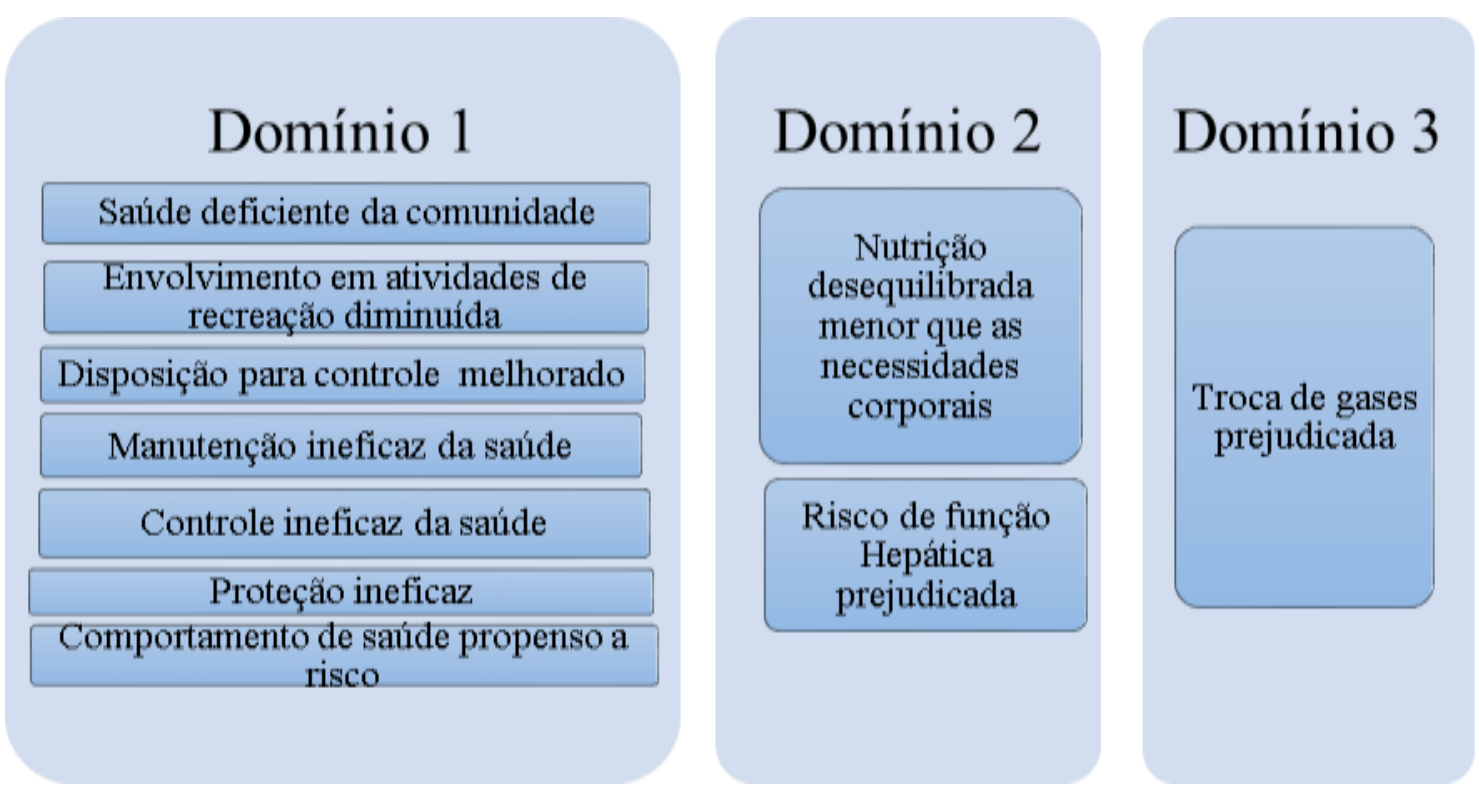

Fonte: Autores (2020). 
Figura 2. Lista de Diagnósticos associados aos COVID-19, domínios 4 e 5.

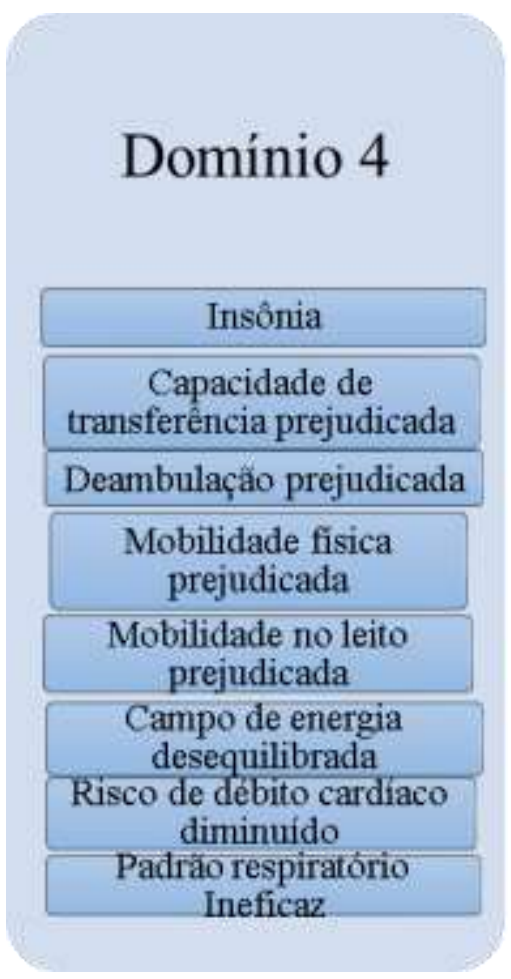

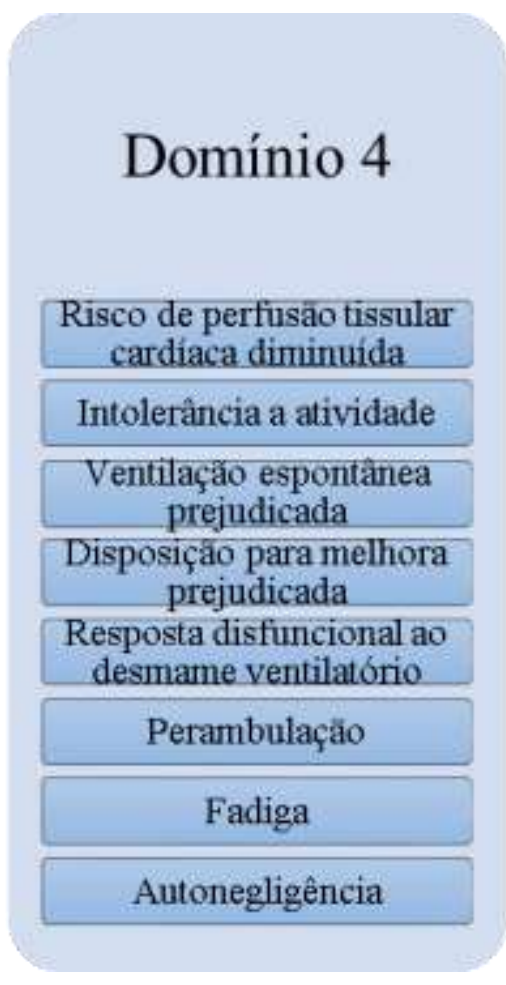

Fonte: Autores (2020).

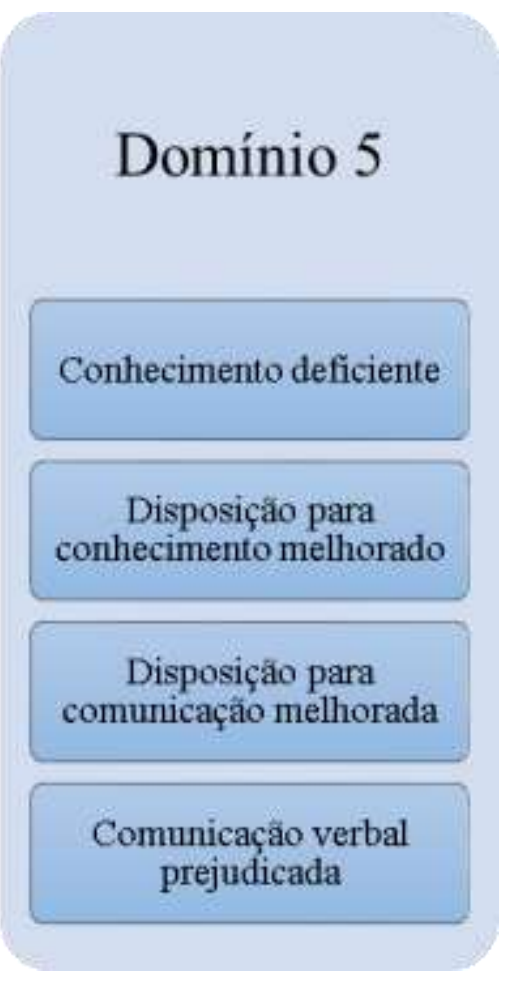

Figura 3. Lista de Diagnósticos associados aos COVID-19, domínios 6,9, 11 e 12.
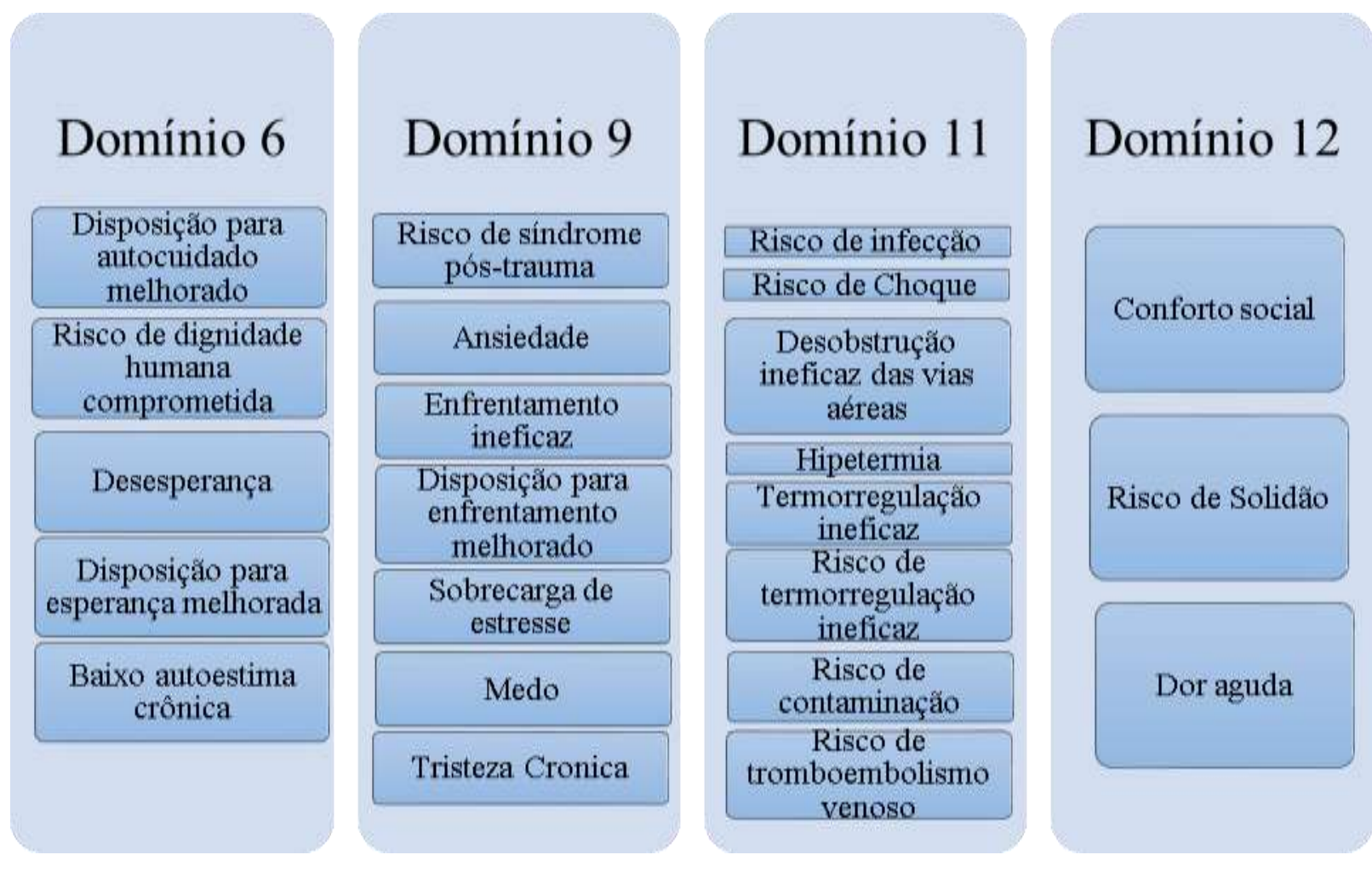

Fonte: Autores (2020). 
Do início foi elaborado um plano com cerca de 40 diagnósticos inicias separados pelos seus respectivos domínios. Os domínios foram divididos por cores, vermelho domínio 1, verde domínio 3, azul domínio 4, laranja domínio 6, rosa domínio 9 e preto domínio 11.

Sabe-se que o processo de enfermagem é um guia sistematizado para um estilo de pensamento que direciona julgamentos clínicos apropriados. Visto isso, é essencial a utilização das taxonomias de enfermagem como a classificação e avaliação, determinação das intervenções e estipular resultados esperados baseados em evidências científicas.

Nos quadros a seguir (Quadros 1 e 2) estão relacionadas as taxonomias para determinar os problemas de enfermagem sugeridas pela North American Nursing Diagnosis Association - NANDA, sendo apresentado os principais diagnósticos de enfermagem para os pacientes acometidos pela infecção pelo Coivd-19. Bem como, as intervenções compatíveis com a atividade laboral de enfermagem, inerentes ao quadro clínico e sintomatologia evidenciada nestes pacientes e de acordo com a Intervention Classification - NIC, permitindo avaliar e nortear estratégias para os enfermeiros implementarem ações efetivas que auxiliem avançar com sucesso do resultado almejado. Além da Nursing Intervention Classification - NOC que trata dos objetivos esperados (Bulechek, 2010; Pereira, 2010; Johnson et al.,2012; Neto et al., 2020).

Quadro1. Diagnóstico de enfermagem, intervenções de enfermagem e resultados almejados relacionados a sintomatologia apresentada pelos pacientes infectados pelo vírus SARS-CoV-2.

\begin{tabular}{|c|c|c|}
\hline NANDA & NIC & NOC \\
\hline $\begin{array}{l}\text { Controle ineficaz de Saúde } \\
\text { relacionado a falha em agir para } \\
\text { reduzir fatores de risco evidencia por } \\
\text { conhecimento insuficiente sobre o } \\
\text { regime }\end{array}$ & $\begin{array}{l}\text { Orientar e ensinar atividades em saúde } \\
\text { para o paciente e a sua família para ter } \\
\text { escolher na vida diária eficaz }\end{array}$ & $\begin{array}{c}\text { Paciente relata fazer escolha da vida } \\
\text { diária eficaz }\end{array}$ \\
\hline $\begin{array}{l}\text { Controle de saúde familiar ineficaz } \\
\text { relacionada a atividades familiares } \\
\text { inadequadas para atingir as metas de } \\
\text { saúde evidenciado por dificuldade de } \\
\text { transitar por sistemas complexos de } \\
\text { cuidados de saúde }\end{array}$ & $\begin{array}{l}\text { Coordenar junto com os órgãos de } \\
\text { saúde e de questões sociais, ações de } \\
\text { saúde comunitária e de controle pelos } \\
\text { órgãos de regulação de sistemas de } \\
\text { saúde. }\end{array}$ & $\begin{array}{l}\text { Paciente relata ter percebido melhora } \\
\text { no controle de saúde familiar. }\end{array}$ \\
\hline $\begin{array}{c}\text { Proteção ineficaz relacionado a } \\
\text { dispneia evidenciado por distúrbios } \\
\text { imunológicos }\end{array}$ & $\begin{array}{l}\text { Trabalhar com oxigenoterapia até } 3 \\
\text { litros e orientar sobre as condições } \\
\text { adequadas de umidade relativa do ar }\end{array}$ & $\begin{array}{c}\text { Paciente terá proteção eficaz de sua } \\
\text { saúde }\end{array}$ \\
\hline $\begin{array}{l}\text { Saúde deficiente da comunidade } \\
\text { relacionada a ausência de programas } \\
\text { para prevenir problemas de saúde de } \\
\text { um grupo ou população evidenciado } \\
\text { por acesso insuficiente a provedores } \\
\text { de cuidados de saúde }\end{array}$ & $\begin{array}{c}\text { Organizar junto a comunidade } \\
\text { deficiente e aos órgãos responsáveis } \\
\text { pela saúde e bem-estar dos mesmos } \\
\text { programas de saúde comunitária. }\end{array}$ & $\begin{array}{c}\text { A comunidade apresenta melhora no } \\
\text { quadro de sua saúde. }\end{array}$ \\
\hline
\end{tabular}

Fonte: Autores (2020). 
Ambas constituem uma linguagem padrão quanto à preconização do uso de determinados termos do processo de Enfermagem, qualificação cuidados e classificação da eficiência da assistência de enfermagem prestada a pacientes, famílias e comunidade. A fim de conhecer melhor as respostas físicas e emocionais reveladas durante o desenvolvimento do processo de trabalho do Enfermeiro (Neto et al., 2020).

Quadro 2. Diagnóstico de enfermagem, intervenções de enfermagem e resultados almejados relacionados a sintomatologia apresentada pelos pacientes infectados pelo vírus SARS-CoV-2.

\begin{tabular}{|c|c|c|}
\hline NANDA & NIC & NOC \\
\hline $\begin{array}{c}\text { Troca de gases prejudicada } \\
\text { relacionada a padrão respiratório } \\
\text { anormal evidenciado por } \\
\text { desequilíbrio na relação ventilação } \\
\text { perfusão }\end{array}$ & $\begin{array}{l}\text { Colocar o paciente em posição de } \\
\text { Fowler e ofertar oxigênio } 3 \text { litros, em } \\
\text { ambiente residencial afastar o paciente } \\
\text { de produtos químicos e manter em } \\
\text { repouso com posição elevada }\end{array}$ & $\begin{array}{l}\text { Paciente apresentará troca de } \\
\text { gases suficiente para manter um } \\
\text { nível adequado a sua saúde. }\end{array}$ \\
\hline $\begin{array}{l}\text { Mobilidade física prejudicada } \\
\text { relacionada a dispneia ao esforço } \\
\text { evidenciado por controle muscular } \\
\text { diminuído }\end{array}$ & $\begin{array}{l}\text { Pacientes receberá aumento da ingesta } \\
\text { hídrica e atividades musculares de } \\
\text { estimulação leve no leito }\end{array}$ & $\begin{array}{l}\text { Paciente apresentara } \\
\text { gradualmente melhora na sua } \\
\text { mobilidade }\end{array}$ \\
\hline $\begin{array}{l}\text { Mobilidade no leito prejudicada } \\
\text { relacionada a capacidade } \\
\text { prejudicada de se mover entre a } \\
\text { posição sentada e a supina } \\
\text { evidenciado por força muscular } \\
\text { insuficiente }\end{array}$ & $\begin{array}{l}\text { Estimulação de movimentação dentro } \\
\text { do leito gradualmente e auxiliar o } \\
\text { paciente em sua mudança de decúbito } \\
\text { de } 2 \text { X } 2 \text { horas }\end{array}$ & $\begin{array}{c}\text { Paciente apresentará mobilidade } \\
\text { no leito adequada }\end{array}$ \\
\hline $\begin{array}{l}\text { Campo de energia desequilibrado } \\
\text { relacionado a diferenciais de } \\
\text { temperatura quente no fluxo } \\
\text { energético evidenciado por doença }\end{array}$ & Verificar sinais vitais de $2 \times 2$ horas & $\begin{array}{l}\text { Paciente restabelece o equilíbrio } \\
\text { do campo de energia }\end{array}$ \\
\hline $\begin{array}{l}\text { Fadiga relacionada a capacidade } \\
\text { prejudicada para manter o nível } \\
\text { habitual de atividade física } \\
\text { evidenciado por exposição a evento } \\
\text { de vida negativo }\end{array}$ & $\begin{array}{c}\text { Orientar a não frequentar ambientes } \\
\text { insalubres, e ter hábitos de vidas } \\
\text { higiênicos e saudáveis }\end{array}$ & Paciente não apresentara fadiga \\
\hline $\begin{array}{l}\text { Perambulação relacionada a } \\
\text { períodos de locomoção intercalados } \\
\text { com períodos de não locomoção } \\
\text { evidenciados por estado fisiológico }\end{array}$ & $\begin{array}{l}\text { Orientar uma perambulação } \\
\text { acompanhado de um profissional ou } \\
\text { acompanhante responsável por um } \\
\text { curto período de tempo e espaço. }\end{array}$ & $\begin{array}{l}\text { Paciente apresentara marchas } \\
\text { frequentes contínuas e firmes, em } \\
\text { um período mais longo }\end{array}$ \\
\hline $\begin{array}{l}\text { Risco de débito cardíaco diminuído } \\
\text { relacionado a alteração no volume }\end{array}$ & $\begin{array}{l}\text { Verificar sinais vitais de } 2 \text { X } 2 \text { horas, } \\
\text { colocar o paciente em decúbito em }\end{array}$ & $\begin{array}{l}\text { Paciente apresentará decúbito } \\
\text { cardíaco normalizado, com o }\end{array}$ \\
\hline
\end{tabular}


Research, Society and Development, v. 10, n. 2, e26410212384, 2021

(CC BY 4.0) | ISSN 2525-3409 | DOI: http://dx.doi.org/10.33448/rsd-v10i2.12384

\begin{tabular}{|c|c|c|}
\hline sistólico & Fowler & $\begin{array}{l}\text { volume sistólico normalizado } \\
\text { dentro dos parâmetros de } \\
\text { normalidades vinculados a OMS }\end{array}$ \\
\hline $\begin{array}{c}\text { Padrão respiratório ineficaz } \\
\text { relacionado a ventilação-minuto } \\
\text { diminuída evidenciado por fadiga da } \\
\text { musculatura respiratória }\end{array}$ & $\begin{array}{c}\text { Colocar o paciente em posição de } \\
\text { Fowler, colocar aporte de oxigênio a } 3 \\
\text { litros e monitorar os sinais vitais } 2 \text { X } 2 \\
\text { horas }\end{array}$ & $\begin{array}{l}\text { Paciente apresentara melhora na } \\
\text { condição do padrão respiratório. }\end{array}$ \\
\hline $\begin{array}{l}\text { Intolerância a atividade relacionada } \\
\text { a desequilíbrio entre a oferta e a } \\
\text { demanda de oxigênio associada por } \\
\text { condição respiratória }\end{array}$ & $\begin{array}{l}\text { Começar a fazer exercícios leves } \\
\text { dentro do leito e deambulação } \\
\text { controlada com tecnologia leve dura de } \\
\text { apoio aplicada }\end{array}$ & $\begin{array}{l}\text { Paciente apresenta melhora para } \\
\text { atividade, sendo mais tolerante a } \\
\text { atividade }\end{array}$ \\
\hline $\begin{array}{l}\text { Ventilação espontânea prejudicada } \\
\text { relacionada a diminuição na } \\
\text { saturação arterial de oxigênio - } \\
\text { SPO2 associada a alteração no } \\
\text { metabolismo }\end{array}$ & $\begin{array}{l}\text { Ofertar oxigênio a } 3 \text { litro, colocar o } \\
\text { paciente na posição de Fowler e } \\
\text { orientar o paciente sobre ansiedade e } \\
\text { controle de inspiração e expiração, } \\
\text { ventilação. }\end{array}$ & $\begin{array}{l}\text { Paciente apresentara melhora na } \\
\text { ventilação espontânea. }\end{array}$ \\
\hline $\begin{array}{c}\text { Disposição para autocuidado } \\
\text { melhorado relacionado a expressa } \\
\text { desejo de melhorar autoconceito } \\
\text { associada por aceitação das } \\
\text { limitações }\end{array}$ & $\begin{array}{c}\text { Orientar sobre o processo da terapia } \\
\text { escolhida e sobre suas demandas e } \\
\text { ações }\end{array}$ & $\begin{array}{l}\text { Paciente apresenta autoconceito } \\
\text { melhorado }\end{array}$ \\
\hline $\begin{array}{l}\text { Risco de dignidade humana } \\
\text { comprometida relacionada a } \\
\text { compreensão insuficiente das } \\
\text { informações de saúde }\end{array}$ & $\begin{array}{l}\text { Orientar e ensinar a respeito de sua } \\
\text { condição clínica, sempre atentando aos } \\
\text { seus sinais e sintomas, mantendo a } \\
\text { calma e a tranquilidade para ganhar a } \\
\text { confiança do paciente }\end{array}$ & $\begin{array}{c}\text { Paciente apresentara compreensão } \\
\text { suficiente sobre informações de } \\
\text { saúde }\end{array}$ \\
\hline $\begin{array}{l}\text { Desesperança relacionado a restrição } \\
\text { prolongada de atividades associada } \\
\text { por desorientação da condição } \\
\text { fisiológica }\end{array}$ & $\begin{array}{l}\text { Orientar e ensinar a respeito de sua } \\
\text { condição clínica, fazendo também } \\
\text { atividades de estimulação dentro do } \\
\text { leito com apoio dos familiares }\end{array}$ & $\begin{array}{l}\text { Paciente consegue se manter } \\
\text { esperançoso. }\end{array}$ \\
\hline $\begin{array}{c}\text { Disposição para esperança } \\
\text { melhorada relacionada a expressa } \\
\text { desejo de aumentar a esperança } \\
\text { associada por expressa desejo de } \\
\text { aumentar a percepção de sentido da } \\
\text { vida }\end{array}$ & $\begin{array}{l}\text { Orientar e ensinar a respeito de sua } \\
\text { condição clínica, sempre atentando aos } \\
\text { seus sinais e sintomas, mantendo a } \\
\text { calma e a tranquilidade para ganhar a } \\
\text { confiança do paciente }\end{array}$ & $\begin{array}{c}\text { Paciente apresentara melhora na } \\
\text { disposição para esperança. }\end{array}$ \\
\hline $\begin{array}{l}\text { Enfrentamento ineficaz relacionado } \\
\text { a resolução insuficiente de } \\
\text { problemas associada por crise } \\
\text { situacional }\end{array}$ & $\begin{array}{l}\text { Orientar e ensinar a respeito de sua } \\
\text { condição clínica e dos métodos } \\
\text { terapêuticos }\end{array}$ & $\begin{array}{c}\text { Paciente apresentará enfretamento } \\
\text { eficaz. }\end{array}$ \\
\hline
\end{tabular}


Research, Society and Development, v. 10, n. 2, e26410212384, 2021

(CC BY 4.0) | ISSN 2525-3409 | DOI: http://dx.doi.org/10.33448/rsd-v10i2.12384

\begin{tabular}{|c|c|c|}
\hline $\begin{array}{c}\text { Disposição para enfrentamento } \\
\text { melhorado relacionado a expressa } \\
\text { desejo de aumentar o uso de } \\
\text { estratégias voltadas ao problema } \\
\text { associada por consciência de } \\
\text { possível mudança ambiental }\end{array}$ & $\begin{array}{c}\text { Orientar e ensinar a respeito de sua } \\
\text { condição clínica e dos métodos } \\
\text { terapêuticos }\end{array}$ & $\begin{array}{c}\text { Paciente aumentara a sua } \\
\text { disposição melhorada para } \\
\text { enfrentamento }\end{array}$ \\
\hline $\begin{array}{c}\text { Medo relacionado a sensação de } \\
\text { pânico evidenciado por cenário } \\
\text { pouco conhecido }\end{array}$ & $\begin{array}{c}\text { Orientar e ensinar a respeito de sua } \\
\text { condição clínica e dos métodos } \\
\text { terapêuticos mantendo a calma e a } \\
\text { tranquilidade para ganhar a confiança } \\
\text { do paciente }\end{array}$ & $\begin{array}{c}\text { Paciente não apresentará medo } \\
\text { relacionado a sensação de pânico }\end{array}$ \\
\hline $\begin{array}{c}\text { Tristeza crônica relacionado a } \\
\text { tristeza evidenciado por crise no } \\
\text { controle da doença }\end{array}$ & $\begin{array}{c}\text { Orientar e ensinar a respeito de sua } \\
\text { condição clínica e dos métodos } \\
\text { terapêuticos mantendo a calma e a } \\
\text { tranquilidade para ganhar a confiança } \\
\text { do paciente }\end{array}$ & $\begin{array}{c}\text { Paciente apresentara melhora no } \\
\text { quadro de tristeza }\end{array}$ \\
\hline $\begin{array}{c}\text { Risco de infecção relacionado a } \\
\text { evitar exposição a patógenos }\end{array}$ & $\begin{array}{c}\text { Orientar e ensinar a respeito de sua } \\
\text { condição clínica e dos métodos } \\
\text { terapêuticos }\end{array}$ & $\begin{array}{c}\text { Risco de infecção para o paciente } \\
\text { fica controlado }\end{array}$ \\
\hline
\end{tabular}

Fonte: Autores (2020).

Os quadros apresentados com os diagnósticos de enfermagem mostraram a relevância do papel do enfermeiro e da equipe de enfermagem mediante aos sinais e sintomas da patologia referida e no enfrentamento à pandemia.

Sabe-se que o processo de enfermagem é um guia sistematizado para um estilo de pensamento que direciona julgamentos clínicos apropriados. Visto isso, é essencial a utilização das taxonomias de enfermagem como a classificação e avaliação, determinação das intervenções e estipular resultados esperados baseados em evidências científicas (JOHNSON, et al., 2009).

O presente estudo teve como finalidade, propor uma formulação de semiologia, elaborando diagnósticos de enfermagem pré-agrupados, traçando estratégias de enfrentamento desta e de outras pandemias que venham surgir, através da relação dos cuidados de enfermagem com o avanço clínico de melhora do paciente.

\section{Considerações Finais}

Os pacientes portadores de Coronavírus 2019-Ncov apresentaram melhora gradual referente aos sinais e sintomas, entretanto ainda esperam uma terapêutica mais eficiente, como por exemplo, sorotipos, recombinados do Coronavírus.

Com o passar do tempo houve o surgimento de novas evidencias acerca do COVID-19, isso foi sendo elaborado de acordo com alastramento de sua contaminação, ou seja, a pandemia, o número de casos global foi aumentando e mostrando que os sistemas de saúde dos países, não estavam preparados, pelo menos e o que o cenário está mostrando.

No Brasil, diferentes medidas têm sido adotadas para tentar conter o aumento do número de infecções por SARSCoV-2. O Ministério da Saúde, por meio de diferentes departamentos, tem reunido esforços no sentido de organizar os serviços de saúde para o atendimento de pacientes com suspeita ou diagnóstico confirmado de COVID-19, bem como de qualificar esse 
atendimento. Entre essas medidas estão o Plano de Contingência Nacional para Infecção Humana pelo novo Coronavírus, coordenado pela Secretaria de Vigilância em Saúde (SVS), o Protocolo de Manejo Clínico do Coronavírus (COVID-19) na Atenção Primária à Saúde, da Secretaria de Atenção Primária à Saúde (SAPS), a Nota Informativa n. 6/2020 DAF/SCTIE/MS, entre outros documentos (MS, 2020).

Com este estudo evidenciamos a necessidade de uma terminologia aplicada para a assistência de enfermagem bem como, os benefícios de sua implementação. Além da identificação dos diagnósticos que mais apareceram no estudo e o porquê de sua implementação. Fundamentamos ainda, a relevância do papel do enfermeiro frente a pandemia de COVI-19, pontuando os cuidados que não podem deixar de ser prestados para que assim obtenha o êxito no cuidado.

Neste contexto, vale ressaltar a grande necessidade de diagnósticos e intervenções de enfermagem no cuidado e na recuperação para as pessoas com COVID-19. Entretanto, por ser recente o aparecimento da cepa do vírus e termos poucos estudos realizados nos deparamos com escassez de artigos sobre a temática apesar de ser um assunto de grande importância.

Apesar de termos uma legislação do Conselho Federal de Enfermagem em vigor desde 2019, que dá obrigatoriedade da utilização de diagnósticos desde então, mostra-se que não se percebe sua aplicabilidade por muitos profissionais que atuam diretamente na prática, conotando a importância de mais publicações acerca do objeto de estudo desta pesquisa.

Com base nessa relação, o estudo estabelece que os diagnósticos referidos anteriormente sejam uma base de critérios clínicos para a enfermagem prestar cuidados aos pacientes acometidos com COVID- 19. Mesmo com o avançar das descobertas científicas a respeito da melhora do cuidado e do tratamento da COVID-19, esta base de diagnósticos de Nanda, ajudará ao enfermeiro, enfermeira e a equipe de enfermagem, a entender o processo desta patologia ao paciente.

O desenvolvimento de um artigo científico contribui para a propagação do conhecimento, uma vez que surge da necessidade de responder à questionamentos e solucionar problemas por meio da aplicação de processos metodológicos, e êxito nos resultados obtidos. A atualização deste conhecimento é gerada em todo o mundo por intermédio de uma rede de colaboração entre cientistas. Tornando-se notório a relevância da produção e publicação de novos artigos científicos acerca das mais variadas temáticas, cuja ação contribui com grande valia não só para a classe acadêmica, mas também, em benefício de toda a sociedade.

Ao realizar estudos científicos futuros, pesquisadores demonstram comprometimento e responsabilidade com a ciência através de suas publicações, em que a principal motivação é a contribuição efetiva no avanço das pesquisas no país, bem como, o reconhecimento, visibilidade dos autores e suas respectivas instituições. Além de incentivar e inspirar outros pesquisadores a explorarem novos aspectos sobre o mesmo tema.

Nesse contexto, no que tange o tema proposto no presente estudo, o papel da ciência tem se mostrado crucial neste momento de pandemia, onde o esforço e união de cientistas em prol de uma única causa, fez com que aumentasse exponencialmente a credibilidade e a confiança da população na ciência, fomentada por trabalhos importantes elaborados por Universidades públicas do Brasil com estratégias e ações no combate ao Covid-19.

\section{Referências}

Bai, Y. (2020). Presumed Asymptomatic Carrier Transmission of COVID-19. Paraná: JAMA.

Bangalore, S., Sharma, A., Slotwiner, A. (2020). ST-Segment Elevation in Patients with Covid-19 - A Case Series. $N$ Engl J Med, Apr 17

BBC NEWS - BRASIL (2020) Coronavírus: estudo revela fatores de risco e sintomas em pacientes mortos em hospital na China. Recuperado de: https://www.bbc.com/portuguese/geral-51810488

BRASIL. MINISTÉRIO DA SAÚDE (2020) Protocolo de manejo clínico para o novo-coronavírus (2019-nCoV). Recuperado de: https://portalarquivos2.saude.gov.br/images/pdf/2020/fevereiro/11/protocolo-manejo- -coronavirus.pdf. 
Bulechek, G. M; Butcher, H. K; Dochterman, J. M. Classificação das intervenções de enfermagem (NIC). [tradução Soraya Imon de Oliveira... et al]. - $5^{\text {a }}$ ed. Rio de Janeiro: Elsevier, 2010. Disponível em: https://www.biosanas.com.br/uploads/outros/artigos_cientificos/14/0ac4055be9a07e3df54c72e9651c589e.pdf Acessado em: 06 de fevereiro de 2021

Cespedes, M. S.; Souza, J. C. R. P. (2020) Coronavirus: a clinical update of Covid-19. Rev Assoc Med Bras; 66(2):116-123. Recuperado de: https://blog.scielo.org/wp-content/uploads/2020/04/1806-9282-ramb-66-2-0116.pdf

Doremalen, N., Bushmaker, T., Morris, D., Phil, M., Holbrook, M., Gamble, A., Williamson, B., ... Muster, V. (2020). Aerosol and Surface Stability of SARSCoV-2 as Compared hith SARS-CoV1. NEJM, 382, 16. Recuperado de: http://www.toledo.ufpr.br/portal/artigos-cientificos-covid-19/

Johnson, M. (2012). Ligações NANDA - NOC - NIC: condições clínicas: suporte ao raciocínio e assistência de qualidade. [et al.; tradução de Soraya Imon de Oliveira... et al.]. - $3^{\mathrm{a}}$ ed. Rio de Janeiro: Elsevier, 2012. Disponível em: http://www.faesb.edu.br/biblioteca/wp-content/uploads/2020/03/Ligacoes-entreNANDA-NOC-e-NIC-Marion-Joh.pdf

Acessado em: 06 de fevereiro de 2021.

Johnson, M. (2021). Ligações entre NANDA-NIC-NOC, diagnósticos, resultados e intervenções de enfermagem. $2^{\mathrm{a}}$ ed. Porto Alegre (RS): Artmed; 2009. Disponível em: http://www1.udesc.br/arquivos/id_submenu/2259/2.pdf._Acessado em: 06 de fevereiro de 2021.

Hu, B., Ge, X., Wang, L. F., \& Shi, Z. (2015). Bat origin of human coronaviruses. Virology journal, 12, 221. https://doi.org/10.1186/s12985-015-0422-1

Inui S., Fujikawa A., Jitsu M., Kunishima N., Sadahiro W., Suzuki Y., Umeda S., \& Uwabe Y. (2020). Chest CT Findings in Cases from the Cruise Ship "Diamond Princess" with Coronavirus Disease 2019 (COVID-19). RSNA, 1-17. https://doi.org/10.1148/ryct.2020200110

Lima, C. M. A. O. (2020). Information about the new coronavirus disease (COVID-19). Radiologia Brasileira, 53(2), V-VI. Epub. https://doi.org/10.1590/0100-3984.2020.53.2e1

Madjid, M., Safavi-Naeini, P., Solomon, SD., \& Vardeny, O. (2020). Potential Effects of Coronaviruses on the Cardiovascular System: A Review. JAMA Cardiol. https://doi: 10.1001/jamacardio.2020.12864

Matt, A., Yim, E., Klaff, L., Lokhandwala, S., Riedo, M. F., Chong, M., \& Lee, M. (2020). Characteristics and outcomes of 21 critically Ill patients with COVID-19 in Washington State. JAMA. Published online March 19, 2020. Disponível em: toledo.ufpr.br. Doi: 10.1001/jama.2020.4326

NANDA. Diagnósticos de enfermagem da NANDA-I. Definições e classificações. 2018. Porto Alegre, BR. Recuperado de: https://edisciplinas.usp.br/pluginfile.php/4976902/mod_resource/content/1/NANDA-I-2018_2020.pdf.

Neto (2021). Diagnósticos/resultados e intervenções de enfermagem para pacientes graves acometidos por covid-19 e sepse. Texto \& Contexto Enfermagem, v. 29: e20200160, 2020. Disponível em: https://www.scielo.br/pdf/tce/v29/pt_1980-265X-tce-29-e20200160.pdf. DOI https://doi.org/10.1590/1980-265XTCE-2020-0160._Acessado em: 06 de fevereiro de 2021.

Nobre, A., Sousa, R., Santos, M., Barbagelata, L., Junior, E., Lima, D., ... Mello, W. (2014). Primeira detecção de coronavírus humano associado à infecção respiratória aguda na Região Norte do Brasil. Revista Pan-Amazônica de Saúde, 5(2), 37-41. Recuperado de: http://scielo.iec.gov.br/scielo.php?script=sci_arttext\&pid=S2176-62232014000200005\&lng=pt\&tlng=pt.

Pereira A.S. et al. (2018). Metodologia da pesquisa científica. [e-book]. Santa Maria. Ed. UAB/NTE/UFSM. Recuperado de: em: https://repositorio.ufsm.br/bitstream/handle/1/15824/Lic_Computacao_Metodologia-Pesquisa-Cientifica.pdf?sequence=1

Pereira, J. C; StuchI, R. A. G; Sena, C. A. Proposta de Sistematização da Assistência de Enfermagem pelas Taxonomias NANDA/NIC/NOC para o Diagnostico de Conhecimento Deficiente. Cogitare Enferm. Jan/Mar; 15(1):74-81, 2010.2 Disponível em: file:///D:/Users/Cristiane/Desktop/TRABALHO\%20COVID19/ARTIGOS/taxonomia\%20de\%20nanda.pdf._Acessado em: 06 de fevereiro de 2021.

Siegel, M.; Parsons, P.; Finlay, G. (2020) Síndrome do desconforto respiratório agudo: epidemiologia, fisiopatologia, patologia e etiologia em adultos. UpToDate. March 24, 2020. Recuperado de: http://www.toledo.ufpr.br/portal/artigos-cientificos-covid-19/

Tang, N., Li, D., Wang, X. (2020) Abnormal coagulation parameters are associated with poor prognosis in patients with novel coronavirus pneumonia. Journal of Thrombosis and Hemostasis, 18:844-847, 2020. https://doi:10.1111/jth.14768

Tang, N., Bai, H., Chen, X., Gong, J., Li, D., \& Sun, Z. (2020) Anticoagulant treatment is associated with decreased mortality in severe coronavirus disease 2019 patients with coagulopathy. Journal of Thrombosis and Hemostasis, 27, 2020. https://doi.org/10.1111/jth.14817

Wertheim, J. O. (2013) A Case for the Ancient Origin of Coronaviruses. Journal of Virology. June, Vol 87 Number 12 p. 7039 -7045, 2013. ISSN 0022538X. PMC 3676139 . PMID 23596293. https://doi:10.1128/JVI.03273-12

Woo, P. C. Y. (2009) Coronavirus diversity, phylogeny and interspecies jumping. Exp Biol Med (Maywood). 2009;234(10):1117-1127. https://doi:10.3181/0903-MR-94

Wu, D. (2020). The SARS-CoV-2 outbreak: what we know. International Journal of Infectious Diseases. Published online March 12, 2020. Recuperado de: http://www.toledo.ufpr.br/portal/artigos-cientificos-covid-19/

Yuen K., Ye Z., Fung S., Chan C., \& Jin D. (2020). SARS-CoV-2 and COVID-19: The most important research questions. Cell \& Bioscience, 10, 40. https://doi.org/10.1186/s13578-020-00404-4

Zheng, Y. (2020) COVID-19 and the cardiovascular system. Nature. Published online: March 5, $2020 . \quad$ Recuperado de: http://www.toledo.ufpr.br/portal/artigos-cientificos-covid-19/

Zhou, F. (2020) Clinical course and risk factors for mortality of adult inpatients with COVID-19 in Wuhan, China: a retrospective cohort study. Lancet, March 11, 2020. https://doi.org/10.1016/50140-6736(20)30566-3. 